Erschienen in: Blühdorn, Hardarik/Breindl, Eva/Waßner, Ulrich Hermann (Hrsg.): Text - Verstehen. Grammatik und darüber hinaus. - Berlin/New York: de Gruyter, 2006. S. 108-124.

(Jahrbuch des Instituts für Deutsche Sprache 2005)

\author{
MAREK KONOPKA
}

\title{
Topologie komplexer Sätze und Textverstehen Zur Stellung von Verbletztsätzen mit weil
}

\begin{abstract}
Für die Stellung der Nebensätze scheint es oft Alternativen zu geben. So können Verbletztsätze mit weil, die den Subjunktorphrasen zugerechnet werden, verschiedene Positionen in der übergeordneten Konstruktion einnehmen. Zwischen Konstruktionen mit Subjunktorphrasen in verschiedenen Stellungen gibt es allerdings Unterschiede. Diese Unterschiede können den semantischen Bezug der Subjunktorphrase zum Rest der Konstruktion und die Gliederung der Gesamtkonstruktion in kommunikative Minimaleinheiten betreffen. In Sachtexten sind die topologischen Möglichkeiten von Subjunktorphrasen und die Möglichkeiten der informationsstrukturellen Gestaltung der Gesamtkonstruktionen eingeschränkt. Hier können sich für die geschriebene Sprache spezifische Ambiguitäten in Bezug auf die Fokus-Hintergrund-Gliederung und die Gliederung in kommunikative Minimaleinheiten ergeben, was vom Textautor zu bedenken ist.
\end{abstract}

\section{Das Phänomen: Einleitung}

Allgemein bekannt ist das Phänomen, dass Nebensätze gleichen Typs verschiedene Positionen in der übergeordneten Konstruktion einnehmen können. Besondere „Stellungsfreiheit“ wird üblicherweise Nebensätzen bescheinigt, die syntaktisch als Supplement und semantisch als Adverbiale fungieren können. Als prototypisch sind hier komplexe Einheiten zu sehen, die streng genommen keine Sätze sind, sondern Verbindungen aus einem Konnektor und dem durch diesen regierten Verbletztsatz. Konnektoren, die es erlauben, dass solche Verbindungen im übergeordneten Satz an unterschiedlichen Stellen auftreten und vor allem das Vorfeld besetzen können, werden hier mit Pasch et al. (2003) Subjunktoren genannt. Die Konstruktionen aus dem Subjunktor und dem regierten Verbletztsatz werden dementsprechend als Subjunktorphrasen bezeichnet (vgl. Pasch et al. 2003, S. 351 ff.).

Subjunktorphrasen können grundsätzlich im Vorfeld und im Mittelfeld des Bezugssatzes erscheinen oder diesem nachgestellt werden; sie können auch syntaktisch desintegriert vor dem Vorfeld stehen:

(1) Weil er als Schwuler niemals Kinder haben wird, wäre Stapel fast mit einer Frau vorm Standesamt gelandet. (Die Zeit, 28.01.1999, S. 11) Vorfeldstellung 
(2) Zur Aufdeckung solcher unbewußten Ordnungsmuster hat die Ethnologie, weil sie sich als Disziplin mit Integration befaßt, ein vielseitiges Instrumentarium entwickelt. (Die Zeit, 02.06. 1999, S. 45) - Mittelfeldstellung

(3) Bisher stellt das kein Problem dar, weil es die Ausnahme ist. (Die Zeit, 07.01.1999, S. 4) - Nachstellung

(4) Weil Sie gerade vom Lügen sprechen: In „Mickey Blue Eyes“ gefährdet letztlich eine Lüge Ihre Beziehung. (Züricher Tagesanzeiger, 01.11. 1999, S. 53) - syntaktische Desintegration

Diese topologischen Möglichkeiten in authentischen Beispielen mit naturgemäß variierendem Wortmaterial werfen Fragen auf: Inwieweit können Subjunktorphrasen auch in Konstruktionen mit identischem Wortmaterial verschiedene Positionen einnehmen? Welche Unterschiede bestehen dann zwischen den alternativen Konstruktionen? Und schließlich die beiden zentralen Fragen: Was determiniert in einem konkreten Text die Wahl einer bestimmten Position, und welche Konsequenzen hat diese Wahl für das Textverstehen?

Die in (1) bis (4) illustrierten topologischen Möglichkeiten gelten prinzipiell auch für konditionale Verberstsätze und durch Konnektoren wie angenommen, vorausgesetzt regierte Verbzweitsätze. Der begrenzte Umfang dieses Beitrags erlaubt es nicht, auch diese Konstruktionen eingehend zu untersuchen. Ich beschränke mich deshalb weitgehend auf die Subjunktorphrasen als den typischen Fall der so genannten Nebensätze, die positionsvariabel sind, und versuche anhand von weil-Phrasen aus den Ergebnissen bisheriger Forschung einige allgemeine Regeln zur Stellung von Subjunktorphrasen abzuleiten und sie dann im Hinblick auf die geschriebene Sprache unter Verwendung von authentischen Beispielen zu präzisieren. Dabei werden vor allem Konstruktionen betrachtet, in denen die Subjunktorphrase in einen Verbzweit- oder Verberstsatz eingebettet ist bzw. sich auf einen solchen bezieht. Nicht behandelt werden die Linksversetzungskonstruktionen, da sie gegenüber den oben genannten Beispielen grundsätzlich ein zusätzliches Element, das Korrelat, aufweisen und insofern nicht direkt vergleichbar sind. Linksversetzungskonstruktionen mit weil-Phrasen wie (5) sind im Übrigen in Sachtexten selten:

(5) Weil Literatur für ihn ein einzigartiger Gedächtnisspeicher ist, deshalb ist der Autor Lenz bis heute ein besessener Leser geblieben, wie seine umfangreichen Essaybände zeigen. (Die Zeit, 19.03.1998, S. 54)

\section{Syntaktische Desintegration im Vorvorfeld}

Betrachtet man noch einmal die Beispiele (1) bis (4), so ist nicht zu übersehen, dass das letzte aus der Reihe fällt. Die Subjunktorphrase steht hier im so genannten Vorvorfeld bzw. nimmt die Nullstelle genannte Position ein und ist nicht eingebettet, das heißt, sie erfüllt in der übergeordneten Konstruktion 
keine syntaktische Funktion. Versucht man Beispiele für verschiedene Stellungen der weil-Phrase mit identischem Wortmaterial zu konstruieren, so wird auch der besondere semantische Status der syntaktisch desintegrierten Subjunktorphrase deutlicher:

(6a) Weil es regnen soll, wollen wir zu Hause bleiben.

(6b) Wir wollen, weil es regnen soll, zu Hause bleiben.

(6c) Wir wollen zu Hause bleiben, weil es regnen soll.

(6d) "Weil es regnen soll, wir wollen zu Hause bleiben.

Drückt die weil-Phrase eine Begründung für die Proposition des Bezugssatzes aus, was als der typische Fall ihrer Verwendung zu sehen ist, so ist sie - wie in (6a) bis (6c) - im Vorfeld, im Mittelfeld und auch nachgestellt möglich. Die syntaktische Desintegration der weil-Phrase in (6d) erscheint hingegen kaum akzeptabel. Vollkommen unproblematisch ist aber eine Konstruktion wie

(7) Weil du es unbedingt wissen willst, wir wollen zu Hause bleiben.

Die weil-Phrase liefert in (7) keine Begründung für die Proposition des Verbzweitsatzes, sondern eine Begründung für dessen Äußerung. Ihre Bedeutung könnte wie folgt paraphrasiert werden: ,Weil du es unbedingt wissen willst, sage ich dir ...' (ähnlich Küper 1991, S. 148f.). Eine vergleichbare, nicht auf die Proposition des Folgesatzes bezogene Interpretation ist für die weil-Phrase in (6d) schwer denkbar, und mit propositionalem Bezug ist sie syntaktisch desintegriert im Vorvorfeld offensichtlich nicht möglich.

Die syntaktische Desintegration im Vorvorfeld ist typisch für Subjunktorphrasen, die wie in (4) oder (7) als Sprechhandlungskommentar verwendet werden. Zifonun et al. (1997, S. $2303 \mathrm{ff}$.) betrachten diese Verwendung als eine Spielart der Kommentierung des Modus Dicendi. Entsprechend verwendete Subjunktorphrasen werden in der Literatur auch als Sprechaktadverbiale oder pragmatische bzw. illokutive Adverbiale bezeichnet (Pittner 1999, S. 320).

Was die syntaktische Desintegration im Vorvorfeld im Allgemeinen betrifft, so ist dabei zu beobachten, dass der auf den desintegrierten Ausdruck folgende Satz immer eine kommunikative Minimaleinheit (Zifonun et al. 1997, S. 86ff.) bildet. Bereits mit ihm allein wird eine sprachliche Handlung vollzogen. Syntaktisch desintegrierte Ausdrücke sind insofern als metakommunikative Kommentare zu betrachten (vgl. Pasch et al. 2003, S. 266). Semantisch gesehen erscheint die syntaktische Desintegration einer Subjunktorphrase im Vorvorfeld insbesondere dann möglich, wenn der Bezugssatz fokussiert ist und die Subjunktorphrase zu seinen Wahrheitsbedingungen nichts beiträgt (zu letzterer und weiteren Voraussetzungen siehe König/van der Auwera 1988, S. 110 ff.; vgl. auch Peyer 1997, S. 86 ff.; Zifonun et al. 1997, S. 2350f.; zu gesprochener Sprache teilweise abweichend Auer 1997, S. 67-74). Die syntaktische Desintegration erscheint dementsprechend regulär, wenn die Subjunktorphrase ein Irrelevanzkonditional ist (vgl. (8) unten), und häufig, wenn die 
Subjunktorphrase ein Konzessivausdruck ist (vgl. (9) unten) - in diesen beiden Fällen ist der Bezugssatz in seinen Wahrheitsbedingungen immer von der Subjunktorphrase unabhängig; möglich ist sie aber auch bei konditionalen, finalen, temporalen und - wie bereits gezeigt - kausalen Subjunktoren (Pasch et al. (2003, S. 392) liefern eine Liste der relevanten Subjunktoren).

(8) Mit dieser Ausgabe wechselt die ZEIT von der alten zur neuen Rechtschreibung. Warum? Ob sie einem nun mehr oder weniger oder gar nicht gefällt, sie wird bald eine Selbstverständlichkeit sein. (Die Zeit, 10.06. 1999, S. 37)

(9) Obwohl der detailsüchtige Autor immer wieder Jahreszahlen nennt, längst Vergangenes beschwört: Die Geschichte, die so gelassen erzählt wird, kommt mir nicht als historisches Geschehen vor. (Die Zeit, 04. 02. 1999, S. 42)

Metakommunikative Subjunktorphrasen können zuweilen auch parenthetisch in die Linearstruktur des Bezugssatzes eingeschoben oder nachgestellt als Nachtrag realisiert werden. In diesen Fällen kann die prosodische Herausgehobenheit der Subjunktorphrase als ein Indiz für ihren besonderen Status gewertet werden, allerdings kein besonders zuverlässiges, denn prosodisch desintegriert können auch fokussierte Subjunktorphrasen mit propositionalem Bezug sein (vgl. weiter unten). Schließlich erscheinen metakommunikative Subjunktorphrasen manchmal auch eingebettet, vor allem wenn sie sich explizit oder formelhaft auf die Sprechhandlung beziehen. Man vergleiche etwa das folgende Beispiel mit der Vorfeldposition einer wenn-Phrase:

(10) Wenn Sie mich fragen, wären Nike und Eva Wagner das beste Team, wie damals Wieland und Wolfgang Wagner. (Die Zeit, 12.05. 1999, S. 49)

Gegen die Einbettung von Subjunktorphrasen, mit denen nur implizit Bezug auf die Sprechhandlung genommen wird, spricht, dass sie bei ihrer syntaktischen Integration auch propositionsbezogen interpretiert werden könnten. In diesem Fall wirkt die syntaktische Desintegration desambiguierend (vgl. Thim-Mabrey 1988, S. 52; Pittner 1999, S. 360). Man vgl. etwa das Beispiel

(11a) Weil die Kinder etwas zu essen brauchen, ist der Kühlschrank voll.

in dem die weil-Phrase propositionsbezogen interpretiert werden kann, mit

(11b) Weil die Kinder etwas zu essen brauchen: der Kühlschrank ist voll.

mit der Paraphrase: ,Weil die Kinder etwas zu essen brauchen, sage ich dir: der Kühlschrank ist voll.

Somit erscheint die syntaktische Desintegration im Vorvorfeld für metakommunikative Subjunktorphrasen nicht nur als typisch, sondern auch als die einzige Stellungsmöglichkeit, in der sie ohne zusätzliche Indizien zuverlässig als metakommunikative Kommentare interpretiert werden können. 


\section{Vorfeldstellung, Mittelfeldstellung und Nachstellung und die Fokus-Hintergrund-Gliederung der Gesamtkonstruktion}

Die Vorfeldstellung, die Mittelfeldstellung und die Nachstellung sind die Positionen, in denen, wie es die Beispiele (1) bis (3) und (6a) bis (6c) zeigten, auf jeden Fall Subjunktorphrasen mit propositionalem Bezug auftreten können. Als einer der Faktoren, welche die Wortstellung regulieren, wird immer wieder die Fokus-Hintergrund-Gliederung genannt. So würde man erwarten, dass es zwischen den genannten Stellungsmöglichkeiten Unterschiede in Bezug auf die Fokus-Hintergrund-Gliederung gibt. Unter Fokus werden hier, wie in der Literatur üblich (Pasch et al. 2003, S. $123 \mathrm{ff}$.; vgl. auch etwa Jacobs 1988), diejenigen Anteile an der Bedeutung eines komplexen Ausdrucks verstanden, die mit anderen denkbaren mentalen Einheiten kontrastiert werden. Die Ausdrücke, mit denen diese Bedeutungsanteile realisiert werden, werden als fokussiert betrachtet. Das, was an der Bedeutung eines komplexen Ausdrucks nicht mit etwas anderem kontrastiert werden soll, wird zu dessen Hintergrund gezählt. Im Hinblick auf die behandelten Konstruktionen werden ihre Teilsätze immer als Ganzheiten betrachtet. Das heißt, dass Teilsätze, die als fokussiert bezeichnet werden, neben fokussierten Ausdrücken auch Hintergrundausdrücke enthalten können.

Vergleicht man Subjunktorphrasen-Konstruktionen mit Konstruktionen mit eingeleiteten Verbzweitsätzen oder konditionalen Verberstsätzen, zeigen die ersten die größten Freiheiten in Bezug auf die möglichen Fokus-Hintergrund-Gliederungen. In Konstruktionen mit eingeleiteten Verbzweitsätzen wie Vorausgesetzt, das Wetter stimmt, komme ich mit zum Ausflug sind normalerweise beide Teilsätze unabhängig von ihrer Anordnung gleichzeitig fokussiert, und auch konditionale Verberstsätze können nicht immer die Hintergrundinformation ausdrücken. Fraglich bzw. nicht möglich sind z. B.:

(12) [A: Morgen wird es regnen. B.:] 'Regnet es morgen, komme ich nicht mit zum Ausflug.

(13) [A: Morgen wird es regnen. B.:] Das ist ja auch bitter nötig. Allerdings komme ich nicht mit zum Ausflug, "regnet es morgen. (vgl. Pasch et al. 2003, S. 371 u. 450 f.)

Konstruktionen mit Subjunktorphrasen weisen dagegen (zumindest in gesprochener Sprache) prinzipiell keine Einschränkungen bezüglich des Anteils der Teilsätze an der Fokus-Hintergrund-Gliederung auf (vgl. Pasch et al. 2003, S. 245 u. 371-377): Jede der in (6a) bis (6c) illustrierten Anordnungen in Konstruktionen mit weil-Phrasen erlaubt, dass beide Konnekte des Subjunktors gleichzeitig fokussiert sind oder dass eines von beiden, und zwar ein beliebiges, im Hintergrund ist. Dies illustrieren folgende Beispiele (die fokussierten Ausdrücke sind fett hervorgehoben, der Vokal der Silbe mit dem Hauptakzent ist unterstrichen): 
(14) [A: Morgen soll es regnen. B:]

(14a) Weil es regnen soll, wollen wir zu Hause bleiben.

(14b) Wir wollen, weil es regnen soll, zu Hause bleiben.

(14c) Wir wollen zu Hause bleiben, weil es regnen soll.

(15) [A: Warum wollt ihr zu Hause bleiben? B:]

(15a) Weil es regnen soll, wollen wir zu Hause bleiben.

(15b) Wir wollen, weil es regnen soll, zu Hause bleiben.

(15c) Wir wollen zu Hause bleiben, weil es regnen soll.

(16) [A: Was macht ihr morgen? B:]

(16a) Weil es regnen soll, wollen wir zu Hause bleiben.

(16b) Wir wollen, weil es regnen soll, zu Hause bleiben.

(16c) Wir wollen zu Hause bleiben, weil es regnen soll.

(Die einzelnen Varianten kommen selbstverständlich mit unterschiedlicher Häufigkeit vor, worauf weiter unten noch eingegangen wird.) Möglich ist in solchen Konstruktionen auch, dass beide Konnekte im Hintergrund sind und nur der Subjunktor fokussiert wird (vgl. Pasch et al. 2003, S. 374f.). Dieser Fall ist allerdings recht selten und soll im Folgenden nicht behandelt werden. Die oben präsentierten Konstruktionen werden bis auf (16c) typischerweise jeweils mit einer einzigen Intonationskontur realisiert. Dementsprechend bilden sie eine kommunikative Minimaleinheit mit einer Fokus-HintergrundGliederung, vgl. z. B. (15c):

(15c) [A: Warum wollt ihr zu Hause bleiben? B:] Wir wollen zu Hause bleiben $\uparrow$, weil es regnen soll.

Beispiel (16c), in dem die Subjunktorphrase nachgestellt ist und beide Teilsätze fokussiert sind, zeigt eine Ausnahme. In solchen Konstruktionen werden der vorangehende Satz und die Subjunktorphrase typischerweise jeweils mit einer eigenen Intonationskontur realisiert. Das heißt, dass in einem solchen Fall nach dem ersten Konnekt eine Pause vorliegen kann sowie dass das erste Konnekt mit fallender oder schwebender Intonationskontur endet und die Intonationskontur der Subjunktorphrase höher einsetzt. Die Hauptakzente der beiden Teilsätze werden in jedem Fall nicht zu einer Akzentstruktur integriert (Pasch et al. 2003, S. 392f.). Auf diese Weise wird die Subjunktorphrase prosodisch desintegriert. Es entstehen somit zwei kommunikative Minimaleinheiten mit jeweils eigener Fokus-Hintergrund-Gliederung:

(16c) [A: Was macht ihr morgen? B:] Wir wollen zu Hause bleiben $\downarrow$, weil es regnen soll.

Nachgestellte Subjunktorphrasen in Konstruktionen, in denen beide Teilsätze fokussiert sind, werden nicht dem Nachfeld zugerechnet, sondern als Nachträge betrachtet. In der Literatur werden sie auch als nicht restriktive Nebensätze bezeichnet (z. B. Brandt 1990, S. 72 ff.). Da sie sich nicht an der kommunikativen Minimaleinheit beteiligen, die mit dem vorangehenden Satz gebildet wird, befindet sich ihre Bedeutung auch außerhalb des Skopus von Operato- 
ren, die im ersten Teilsatz auftreten (vgl. Pasch et al. 2003, S. 394; Küper 1991, S. 139):

(17a) [A: Was macht ihr morgen? B:] Vielleicht/Wahrscheinlich bleiben wir zu Hause $\downarrow$, weil es regnen soll. (Modaladverbial)

(17b) [A: Was macht ihr morgen? B:] Wir bleiben nur zu Hause $\downarrow$, weil es regnen soll. (Fokuspartikel)

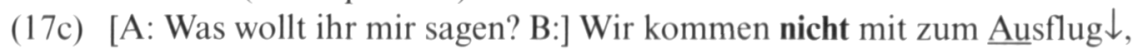
weil es regnen soll. (Negationspartikel)

Dagegen kann sich die Bedeutung der Subjunktorphrase im Skopus eines Operators aus dem ersten Teilsatz befinden, wenn, wie weiter oben in (15c), die Gesamtkonstruktion eine einzige kommunikative Minimaleinheit bildet, vgl.:

(18a) [A: Warum bleiben sie morgen zu Hause? B:] Vielleicht/Wahrscheinlich bleiben sie (deshalb) zu Hause $\uparrow$, weil es regnen soll.

(18b) [A: Warum kommt ihr nicht mit zum Ausflug? B:] Wir würden gerne mitkommen. Wir bleiben nur (deshalb) zu Hause $\uparrow$, weil es regnen soll.

(18c) [A: Ihr bleibt zu Hause, weil es regnen soll? B:] Wir bleiben nicht (deshalb) zu Hause $\uparrow$, weil es regnen soll. [Wir bleiben zu Hause, weil unsere Tochter krank ist.]

(18a) bis (18c) zeigen gleichzeitig, dass in das erste Konnekt ein Korrelat wie deshalb oder deswegen eingefügt werden kann, wodurch eine semantisch äquivalente attributive Korrelatkonstruktion entsteht. Dies gilt für alle Konstruktionen vom Typ (15c). Die Einfügung eines Korrelats bei Fokussierung beider Teilsätze und Realisierung der Subjunktorphrase als Nachtrag ist dagegen nicht möglich, vgl.:

(15c') [A: Warum wollt ihr zu Hause bleiben? B:] Wir wollen deshalb zu Hause bleiben, weil es regnen soll.

(16c') [A: Was macht ihr morgen? B:] "Wir wollen deshalb zu Hause bleiben, weil es regnen soll.

Die Subjunktorphrase wird bei ihrer prosodischen Desintegration in Konstruktionen vom Typ (16c) als nicht eingebettet, also syntaktisch nicht integriert betrachtet. Hier wird mit Pasch (1983, S. 125 ff.; Pasch et al. 2003, S. 395 f.) davon ausgegangen, dass sie als Ellipse zu interpretieren ist. Der Satz, in den sie einzubetten wäre, kann mithilfe des Kontexts rekonstruiert werden. Im Falle von Subjunktorphrasen mit propositionalem Bezug wie den bisher betrachteten kann angenommen werden, dass dem weggelassenen Ausdruck eine Proposition zugrunde liegt, die der des tatsächlich vorangehenden Satzes entspricht, aber nicht fokussiert, sondern im Hintergrund ist. ${ }^{1} \mathrm{Im}$ folgenden Beispiel sind die fokussierten Teile der Konstruktionen fett hervorgehoben:

1 Eine Erklärung, wie die Verknüpfung zwischen dem vorangehendem Satz und der Subjunktorphrase als Nachtrag kognitiv geleistet wird, wird von Konerding (2002, S. 92f.) versucht. 
(16c") [A: Was macht ihr morgen? B:] Wir wollen zu Hause bleiben $\downarrow$. Wir wolten zu Hause bleiben $\uparrow$, weil es regnen soll.

Die kommunikative und syntaktische Abkoppelung der Subjunktorphrase als Nachtrag ermöglicht es, dass sie sich nicht mehr nur auf die Proposition des vorangehenden Satzes beziehen muss. So sind prosodisch desintegriert zum einen metakommunikative Kommentare zulässig, z. B.:

(19) Wir wollen zu Hause bleiben - weil du es sicher wissen willst.

Zum anderen sind als Nachtrag und, ohne dass es zusätzlicher Einschränkungen bedarf, nur als Nachtrag weil-Phrasen mit epistemischem Bezug semantisch akzeptabel; solche weil-Phrasen drücken Begründungen der Sprechereinstellung zur Geltung des Sachverhalts aus, der im Bezugssatz bezeichnet wird, wie z. B. beim reduktiven Schluss in (20a):

(20a) Es hat geregnet $\downarrow$, weil die Straßen nass sind.

Paraphrase: Die Nässe der Straße ist der Grund für die Annahme, dass es geregnet hat.

(20b) " Es hat geregnet $\uparrow$, weil die Straßen nass sind.

(20c) ?"Weil die Straßen nass sind, hat es geregnet.

(20d) "Es hat, weil die Straßen nass sind, geregnet.

Bevorzugte Interpretation für (20b)-(20d): Die Nässe der Straße ist der Grund dafür, dass es geregnet hat.

Bei Fokussierung beider Konnekte des Subjunktors können vor allem umgangssprachlich anstelle von prosodisch desintegrierten weil-Phrasen mit Verbletztsätzen prinzipiell auch nachgestellte Konstruktionen aus weil und so genannten Hauptsätzen (vor allem Verbzweitsätzen) verwendet werden, da die fokussierte Information prototypisch mit Verbzweitsätzen und Verberstsätzen ausgedrückt wird. Dies verdeutlicht, dass der Subjunktor mit dem nachfolgenden Satz eine selbständige kommunikative Minimaleinheit bildet. Während mit der Verwendung eines Verbletztsatzes prinzipiell nicht ausgeschlossen werden kann, dass seine Bedeutung als dem Hintergrund zugehörig interpretiert wird bzw. präsupponiert wird, kennzeichnet ein Verbzweitsatz den bezeichneten Sachverhalt eindeutig als fokussiert und behauptet (vgl. Pasch et al. 2003, S. $407 \mathrm{ff}$.):

$\left(16 c^{\prime \prime \prime}\right)$ Wir wollen zu Hause bleiben, weil: es soll regnen.

(19') Wir wollen zu Hause bleiben, weil: das willst du doch sicher wissen. (vgl. Küper 1991, S. 149)

(20a') Es hat geregnet, weil: die Straßen sind nass.

Vor allem in der Schriftsprache tritt anstelle von weil in Konstruktionen mit nachfolgendem Hauptsatz denn auf, das immer kommunikative Minimaleinheiten verknüpft und somit den Bezug des nachfolgenden Satzes auf nicht propositionale Ebenen der Bedeutung der vorangehenden kommunikativen Minimaleinheit ermöglicht, z. B.: 
(21) Wie bei Bergman oder Ibsen kennt auch dieses Familiendrama keine klassischen Helden, denn es verweigert jene Distanz, aus der heraus die Guten und die Bösen sich ausmachen ließen. (Die Zeit, 11.03.1999, S. 61)

(22) Wir wollen zu Hause bleiben: denn das willst du doch sicher wissen.

An dieser Stelle soll im Hinblick auf die folgenden Ausführungen zweierlei festgehalten werden. Erstens: Bei Nachstellung der Subjunktorphrase mit weil bildet die Gesamtkonstruktion, je nachdem, ob nur ein Teilsatz fokussiert ist oder beide, eine oder zwei kommunikative Minimaleinheiten. Zweitens: Soll eine Konstruktion, in der beide Teilsätze fokussiert sind, eine einzige kommunikative Minimaleinheit bilden, darf die Subjunktorphrase mit weil nicht nachgestellt sein.

\section{Gebrauch der Vorfeldstellung, Mittelfeldstellung und Nachstellung in Sachtexten}

Die im Weiteren zitierten authentischen Beispiele für den Gebrauch der verschiedenen Stellungsmöglichkeiten für Subjunktorphrasen mit weil in Sachtexten stammen aus der Zeitung Die Zeit. Näher untersucht wurden Subjunktorphrasen in den Ausgaben vom Januar bis Juli 1999, aus denen die relevanten Textabschnitte mithilfe von Cosmas II (vgl. http://www.idsmannheim.de/cosmas2/, 29.04.2005) extrahiert wurden.

Für Texte wie die untersuchten gelten drei allgemeine Regularitäten, die vor der Besprechung der Beispiele für übliche Stellungsmöglichkeiten behandelt werden sollen. Sie betreffen die unterschiedliche Häufigkeit, mit der Konstruktionen auftreten, die jenen in (14) bis (16) entsprechen:

A. Die Mittelfeldstellung der Subjunktorphrase ist in Sachtexten wie den untersuchten sehr selten.

Sie tritt etwa dann auf, wenn sich die Subjunktorphrase enger, quasi attributiv, auf eine bestimmte Konstituente des Satzes bezieht (vgl. (23)) bzw. eine Zusatzinformation liefert, die nach der Interpretation der Gesamtkonstruktion für die Textfortsetzung keine Rolle mehr spielt (vgl. (24)). Der Subjunktorphrase folgen in jedem Fall fokussierte Konstituenten des Satzes, die bei Textfortsetzung wieder aufgenommen werden.

(23) Städte und Gemeinden haben schon immer Fremde aufgenommen, auch bevor 1913 die deutsche Staatsangehörigkeit eingeführt wurde. Die einheimischen Vereinbarungen wurden allerdings nicht als Gesetzestexte formuliert und aufgeschrieben, sondern unbewußt getroffen und gelebt $[\ldots]$.

Zur Aufdeckung solcher unbewußten Ordnungsmuster hat die Ethnologie, weil sie sich als Disziplin mit Integration befaßt, ein vielseitiges 
Instrumentarium entwickelt. Dazu zählt insbesondere ihre klassisch gewordene Methode der ,teilnehmenden Beobachtung“. (Die Zeit, 02. 06. 1999, S. 45)

(24) Schließlich konzentrierte ich mich - weil mich ohnehin nichts anderes interessiert - auf die Auskünfte zum Thema Essen. Doch es gab so gut wie keine. (Die Zeit, 10.06.1999, S. 17)

Es ist naheliegend, die Gründe dafür, dass die Subjunktorphrasen kaum im Mittelfeld des Hauptsatzes stehen, in den Besonderheiten der Verarbeitung von Konstruktionen mit Mittelfeldstellung zu suchen: Die Zwischenstellung der Subjunktorphrase erschwert im Allgemeinen die Verarbeitung des Obersatzrestes. Schon in einem unkomplizierten Beispiel wie

Wir wollen, weil es regnen soll, zu Hause bleiben.

ist das „Erkennungsfenster“ für die Bestandteile der relevanten Verbgruppe deutlich größer als in

Wir wollen zu Hause bleiben, weil es regnen soll.

Subjunktorphrasen als Supplementsätze werden aus dem Mittelfeld radikaler verbannt als nomenbezogene Attributsätze. Dies erklärt sich daraus, dass die Mittelfeldstellung nomenbezogener Attributsätze auch einen wesentlichen verarbeitungsrelevanten Vorteil mit sich bringt. Sie bewirkt nämlich gegenüber der Extraposition die Minimierung des Erkennungsfensters für die Bestandteile der Nominalphrase, zu der der Attributsatz gehört. Und dies kann, wenn man hier semantische bzw. informationsstrukturelle Überlegungen bei Seite lässt, den Nachteil der Erweiterung des Erkennungsfensters für die Verbgruppe des Obersatzes aufwiegen (vgl. dazu schon Behaghel 1932, S. $271 \mathrm{ff}$., in jüngerer Zeit z. B. Hawkins 1994, S. 198 ff.).

Sie hat dem Mann, der ihr helfen sollte, den Rücken gekehrt.

Sie hat dem Mann den Rücken gekehrt, der ihr helfen sollte.

Der Komplikation, die sich durch die Mittelfeldstellung der Subjunktorphrase als Supplement ergibt, steht aber kein vergleichbarer Gewinn entgegen. Das scheint ein Grund für eine in Sachtexten geltende stilistische Tendenz, die Mittelfeldstellung der Subjunktorphrase im Allgemeinen zu vermeiden, selbst wenn die Subjunktorphrasen kurz sind. Die Mittelfeldstellung gehört in Sachtexten jedenfalls nicht zu den Hauptstellungsvarianten und wird hier nicht weiter behandelt. 
B. Die Hintergrundinformation bleibt oft unrealisiert oder wird pronominal ausgedrückt.

Die Tendenz zur Eliminierung oder Reduktion der Hintergrundkonnekte ist bereits in gesprochener Sprache deutlich (vgl. Pasch et al. 2003, S. 373). So sind z. B. folgende Reaktionen von B auf die Äußerungen von A üblich, wenn nicht sogar eher zu erwarten als jene in (15) bzw. (14) weiter oben:

(25) [A: Warum wollt ihr zu Hause bleiben? B:] Weil es regnen soll.

(26) [A: Morgen soll es regnen B:] Deshalb wollen wir zu Hause bleiben.

Ähnliche Beispiele sind auch in Sachtexten gang und gäbe, z. B:

(27) Der vierte wollte eine Gewerkschaft für Arbeitslose gründen. In China ist das Aufruf zum Sturz der Staatsgewalt, ersatzweise Hochverrat. Das weiß doch jeder Untertan im Reich der Mitte. Warum steckt dann immer wieder einer seinen Kopf aus der Masse der Gleichgültigen und Geschurigelten?

Weil es in China, wie zu allen Zeiten und an allen Orten, Menschen gibt, die nicht clever genug sein wollen, bei jedem Schritt die Grenze zwischen Kühnheit und Tollkühnheit zu bedenken. (Zeit, 07.01.1999, S. 2)

(28) Joly ist noch nicht verheiratet - und das ist ein Problem. Denn Joly ist bereits 26 Jahre alt. Nicht, daß sich für die hübsche Südinderin kein Mann fände. Doch ihre Familie kann das Geld für die Mitgift nicht aufbringen. Deshalb geht Joly seit sechs Jahren arbeiten. (Die Zeit, 22. 04. 1999, S. 39)

In (27) wird der Bezugssatz der Subjunktorphrase weggelassen, dessen Proposition durch die Frage vorweggenommen wurde, und in (28) erscheint ein Pronominaladverb anstelle einer Subjunktorphrase, deren Proposition der des vorangehenden selbständigen Satzes entsprechen müsste. Es ist davon auszugehen, dass in Sachtexten aufgrund ihres stärker reflektierten Entstehungsprozesses Kommunikationsmaximen wie Sei relevant! oder Sei kurz! (Grice 1979, S. 249 f., vgl. die Diskussion in Schwinn 1997, S. 45 ff.) noch strenger beachtet werden als in gesprochener Sprache, sodass sich die Tendenz zur Eliminierung oder Reduktion der Hintergrundkonnekte unter Umständen sogar verstärkt.

C. Drückt ein Teilsatz Hintergrundinformation aus, so ist zu erwarten, dass er dem fokussierten Teilsatz vorangeht.

Die Voranstellung der Hintergrundinformation kann direkt der Sicherung der Textkohärenz (vgl. auch dazu schon Behaghel 1932, S. 4) und der Motivierung der Textprogression dienen. Die Nachstellung erscheint dagegen kontraintuitiv, weil sie die Textprogression nicht fördert. Daraus resultiert die Tendenz der Leser, den zweiten Teilsatz der Verbindung immer als fokussiert zu interpretieren. In geschriebener Sprache fehlen dem Autor die prosodischen 
Mittel, mit denen er dieser Tendenz entgegenwirken und eventuell den Hintergrundcharakter des nachgestellten Konnekts klarstellen könnte.

Aufgrund der Regularitäten A-C bzw. der zugrundeliegenden Tendenzen sind in Sachtexten wie den untersuchten in Bezug auf die Fokus-HintergrundGliederung nur die folgenden Fälle signifikant:

- Teilsatz mit Hintergrundinformation vor fokussiertem Teilsatz

- beide Teilsätze fokussiert

Dabei kann in beiden Fällen die Subjunktorphrase entweder vorangestellt oder nachgestellt werden. Im zweiten Fall bildet die Gesamtkonstruktion wie weiter oben ausgeführt je nach Stellung der Subjunktorphrase entweder eine oder zwei kommunikative Minimaleinheiten.

Die Besonderheiten der geschriebenen Texte im Hinblick auf die Interpretation der Teilsätze als fokussierte Information oder Hintergrundinformation hängen, wie bereits angedeutet, entscheidend damit zusammen, dass die prosodischen Merkmale der Konstruktionen vom Autor nicht direkt vorgegeben werden können. Vielmehr werden sie - mit letzter Sicherheit erst am Ende der Interpretation - vom Leser rekonstruiert. Die Interpretation der FokusHintergrund-Gliederung hängt vom jeweiligen Leser ab, und zwar in einem nicht zu unterschätzenden Maße, denn jeder Leser baut einen anderen mentalen Kontext auf, mit dem die fokussierten Ausdrücke kontrastieren sollen. Eindeutig dem Hintergrund können in der Regel Teilsätze zugerechnet werden, deren Propositionen unmittelbar rekurrent sind, diese sind allerdings selten. Außerdem sind im Hintergrund Bedeutungsanteile, die evident, inferierbar oder erwartbar sind. Solche Kriterien lassen dem Leser einen großen Spielraum, sodass im gleichen Text ein Teilsatz einmal dem Fokus, ein anderes Mal dem Hintergrund zugerechnet werden kann. Betrachtet werden sollen jedoch zunächst die relativ eindeutigen Beispiele.

In (29) und (30) steht der Teilsatz mit Hintergrundinformation vor dem fokussierten Teilsatz:

(29) zeit: Es gibt schon jetzt annähernd zwei Millionen Menschen mit doppelter Staatsangehörigkeit in Deutschland. Bisher war diese angebliche Privilegierung für den Rest der Bevölkerung kein Problem. Vielleicht wird es das erst mit Ihrer Kampagne.

Schäuble: Bisher stellt das kein Problem dar, weil es die Ausnahme ist. Jetzt aber soll es zum Regelfall werden. $\mathrm{Zu}$ glauben, das ginge ohne öffentliche Erregung ab, wenn sich die CDU/CSU dazu nicht äußern würde, ist absurd. (Die Zeit, 07.01. 1999, S. 4)

(30) ... und die Ski leihen

Wer es einmal getan hat, tut es immer wieder. Mit einer geliehenen Skiausrüstung wird der Winterurlaub viel erholsamer: keine Schlepperei, kein vollgepacktes Auto mit Dachträger, dafür aber jedes Jahr das neueste Modell. Das Ausleihen der Wintersportausrüstung eröffnet außerdem Abwechslung - heute ein Carvingski, morgen ein klassisches 
Modell, übermorgen zur Probe ein Snowboard. Und weil der Skimieter nur halb soviel Gepäck hat, reist er bequem mit dem Flugzeug in die Alpen. (Die Zeit, 07.01. 1999, S. 47)

In (29) ist die Proposition des vorausgehenden Hauptsatzes rekurrent und damit eindeutig im Hintergrund, was in geschriebenen Texten selten vorkommt. Wohlgemerkt, der betreffende Text stellt die Wiedergabe eines Interviews dar. In (30) ist die Proposition der vorangestellten Subjunktorphrase aus dem vorangehenden Kontext leicht erschließbar. Die komplexen Sätze beider Textpassagen würden in gesprochener Sprache mit einer Intonationskontur als eine kommunikative Minimaleinheit realisiert.

In (31) und (32) erscheinen beide Konnekte der Verbindung als fokussiert:

(31) Microsoft hat freilich gute Gründe, sich mit Händen und Füßen dagegen zu wehren, offiziell als Alleinherrscher bezeichnet zu werden. Denn das könnte eine Prozeßwelle gegen den Softwarekonzern auslösen: Weil Konkurrenten vor Gericht nicht mehr extra nachweisen müßten, daß Windows ein Monopol ist, würden sie verstärkt Schadensersatzklagen einreichen. (Die Zeit, 11.03.1999, S. 21)

(32) Dort unten im neuerrichteten Holzmarktbrunnen ging er in der Nacht zum 3. September baden, erzählt Oberbürgermeister Busch. Genau um Mitternacht. Busch hatte eine Wette verloren, weil er nicht glauben wollte, daß in nur 16 Monaten Bauzeit eine Innenstadt neu entstehen kann. (Die Zeit, 07.01. 1999, Nr. 02; Zauber über dem Markt, S. 14)

Die relevante Konstruktion in (31) begründet eine kommunikative Minimaleinheit, die Konstruktion in (32) zwei, das heißt vereinfacht, die letztere Konstruktion müsste gesprochen mit zwei Intonationskonturen realisiert werden.

Dass der erste Teilsatz der Verbindung in geschriebener Sprache eindeutig als fokussiert interpretiert werden kann, ist allerdings nicht allzu häufig (manchem Leser mögen schon bei (31) und (32) Zweifel an der Richtigkeit der vorgestellten Interpretation gekommen sein). Dies liegt daran, dass im ersten Teilsatz der Verbindung verstärkt nach kohärenzsicherndem Material gesucht wird. Die Voranstellung eines bestimmten Teilsatzes kann dann auch dazu genutzt werden, seine Erschließbarkeit aus dem sprachlichen oder nicht sprachlichen Kontext nahe zu legen (vgl. weiter oben sowie Konerding 2002, S. 97-103 vor allem zur kohärenzsichernden Funktion vorangestellter weilPhrasen). Dass der erste Teilsatz der Verbindung hinsichtlich seiner Interpretation als fokussiert oder im Hintergrund befindlich nicht eindeutig bestimmt werden kann, ist jedenfalls eine übliche Situation, vgl.:

(33) Unattraktive Geschäftsbereiche wurden in Joint-ventures abgeschoben oder ganz verkauft. Selbst die traditionsreiche Filmmarke Agfa darf nicht mehr zu Bayer gehören. Weil beim Fotografieren die Elektronik inzwischen viel wichtiger ist als die Chemie, soll Agfa im Sommer selbständig werden. Angenehmer Nebeneffekt des Börsengangs: Zwischen 
6 und 8 Milliarden Mark kommen in die Kasse. (Die Zeit, 04. 03.1999, S. 19)

(34) Heute beschäftigt Solectron fast 29000 Menschen und betreibt weltweit 20 Fabriken. Im vergangenen Quartal verdiente das Unternehmen nach Steuern knapp 64 Millionen Dollar und setzte stolze 2 Milliarden Dollar um. Damit ist es jetzt schon größer als beispielsweise der Computerkonzern Apple.

Der Grund für diese Erfolgszahlen: Solectron kann billiger produzieren, weil es sich nur auf die Herstellung von Hardware konzentriert. Die Fabriken des Unternehmens arbeiten rund um die Uhr. (Die Zeit, 07.01. 1999, S. 21)

Bei (33), wo die Subjunktorphrase vorangestellt ist, kann die Information, dass beim Fotografieren die Elektronik inzwischen viel wichtiger ist als die Chemie, dem Leser bereits aus dem außersprachlichen Kontext bekannt sein oder zumindest erwartbar erscheinen, sie kann auf ihn aber auch als mit seinen Erwartungen kontrastierend wirken. Herauszubekommen, wie sie vom Autor gemeint war, ist schon gar nicht möglich. Dies scheint allerdings ohne größere Auswirkungen auf das Ergebnis des Verstehensprozesses zu sein. Anders verhält es sich mit (34), wo die Subjunktorphase nachgestellt ist. Hier kann die Information, dass Solectron billiger produzieren kann, für manchen Leser bereits aus dem sprachlichen Kontext ableitbar sein und zwar daraus, dass das Unternehmen erfolgreich und größer als andere Unternehmen ist. Bei dieser Interpretation des ersten Teilsatzes befindet er sich im Hintergrund. Die Gesamtkonstruktion bildet eine kommunikative Minimaleinheit und müsste gesprochen mit einer Intonationskontur realisiert werden (vgl. (35a)). Wird die Information des ersten Teilsatzes dagegen, weil nicht unmittelbar aus dem Kontext erschließbar, als neu eingestuft und fokussiert, bildet die Gesamtkonstruktion zwei kommunikative Minimaleinheiten und müsste in gesprochener Sprache mit zwei Intonationskonturen realisiert werden (vgl. (35b)).

(35a) Solectron kann (deswegen) billiger produzieren $\uparrow$, weil es sich nur auf die Herstellung von Hardware konzentriert.

(36b) Solectron kann billiger produzieren $\downarrow$, weil es sich nur auf die Herstellung von Hardware konzentriert.

Die Konstruktionen mit Nachstellung der Subjunktorphrase können folglich beim Fehlen von prosodischen Hinweisen (was auch der Normalfall ist) hinsichtlich ihrer Realisierung als eine oder als zwei kommunikative Minimaleinheiten ambig sein. Der Textautor kann dies auch im Voraus bedenken, und dem Leser die gewünschte Interpretation nahe legen. Will er die Gesamtkonstruktion als eine kommunikative Minimaleinheit verstanden wissen, kann er die Subjunktorphrase voranstellen. In diesem Fall bildet die Gesamtkonstruktion, wie es die Beispiele in (14) bis (16) gezeigt haben, immer eine kommunikative Minimaleinheit. Ist dagegen die Aufspaltung der Gesamt- 
konstruktion in zwei kommunikative Minimaleinheiten vom Textautor gewünscht, kann er sie durch grafische Zeichen wie Gedankenstrich oder Punkt signalisieren.

(37) Danach hatten die beiden eine Chance zur Hochzeit verpaßt: IBM-Chef Lou Gerstner und der Chef des Apple-Verwaltungsrats, Mike Markkula, waren zusammengekommen, um über eine mögliche Übernahme zu sprechen. Doch die Verhandlungen kamen nicht weit, - weil Markkula einen zu hohen Preis verlangte. (Die Zeit, 13.10.1995)

(38) Sie sind das einzige Symphonieorchester, das sich seinen Chefdirigenten wählen darf, demokratisch und souverän. Sie lassen selbst bitten. Denn sie können sich das leisten: Die Berliner Philharmoniker sind das beste Orchester der Welt.

Das wird immer gerne behauptet. Weil es sich sowieso nicht nachprüfen läßt. (Die Zeit, 10.06. 1999, S. 48)

Am Ende dieses Abschnitts soll einiges festgehalten werden: Da in Sachtexten Hintergrundkonnekte selten im Mittelfeld stehen und kaum nachgestellt zu erwarten sind, reduziert sich die Anzahl der relevanten Kombinationen aus möglichen Abfolgen der Konnekte und möglichen Fokus-HintergrundGliederungen. Die Fokus-Hintergrund-Gliederung der Konstruktionen kann teilweise unterschiedlich interpretiert werden, vor allem ist der erste Teilsatz der Verbindung oft ambig in Hinblick darauf, ob er fokussiert ist oder zum Hintergrund gehört. Beim Fehlen von Hinweisen auf die prosodische Realisierung der Konstruktionen ergeben sich daraus Ambiguitäten bei Nachstellung der Subjunktorphrase, und zwar im Hinblick darauf, ob die Gesamtkonstruktionen mit einer oder mit zwei Fokus-Hintergrund-Gliederungen und folglich als eine oder zwei kommunikative Minimaleinheiten zu interpretieren sind. Diesen Ambiguitäten kann unter anderem mithilfe von Interpunktion abgeholfen werden.

\section{Resümee}

1. Weil-Phrasen, die sich nicht auf die Proposition des Bezugssatzes beziehen, und insofern semantisch desintegriert sind, erscheinen auch formal desintegriert. Die metakommunikativen Kommentare werden in der Regel durch die Vorvorfeldstellung syntaktisch desintegriert und die Subjunktorphrasen mit epistemischem Bezug werden, da sie mit einer eigenen Intonationskontur nachgetragen werden, prosodisch desintegriert. Die prosodische Desintegration der Subjunktorphrasen als Nachtrag kann auch als ein Zeichen ihrer syntaktischen Desintegration gewertet werden.

2. Propositionsbezogene Subjunktorphrasen können prinzipiell im Vorfeld und im Mittelfeld erscheinen oder nachgestellt werden und in allen diesen Stellungen entweder fokussiert oder im Hintergrund sein. Die Nach- 
stellung einer weil-Phrase in einer Konstruktion, in der beide Teilsätze fokussiert sind, führt zur Aufspaltung dieser Konstruktion in zwei kommunikative Minimaleinheiten.

3. In Sachtexten reduziert sich die Anzahl der signifikanten Stellungen der Subjunktorphrase und Verteilungen der Fokus-Hintergrund-Gliederung. Beim Fehlen von Hinweisen auf die prosodische Realisierung können die Leser die Fokus-Hintergrund-Gliederung unterschiedlich interpretieren. Bei Nachstellung der Subjunktorphrase kommt es folglich zu Schwankungen im Hinblick auf die Realisierung der Gesamtkonstruktion als eine oder als zwei kommunikative Minimaleinheiten. Solchen Interpretationsschwankungen kann der Textautor - wenn es seiner Intention entspricht - allerdings entgegenwirken.

\section{Literatur}

Auer, Peter (1997): Formen und Funktionen der Vor-Vorfeldbesetzung im gesprochenen Deutsch. In: Schlobinski, Peter (Hg.): Syntax des gesprochenen Deutsch. Wiesbaden: Westdeutscher Verlag. S. 55-92.

Behaghel, Otto (1932): Deutsche Syntax. Eine geschichtliche Darstellung. Band IV. Wortstellung. Periodenbau. Heidelberg: Carl Winters Universitätsbuchhandlung.

Brandt, Margareta (1990): Weiterführende Nebensätze: zu ihrer Syntax, Semantik und Pragmatik. Stockholm: Almqvist \& Wiksell. (Lunder Germanistische Forschungen 57).

Grice, Herbert P. (1979): Logik und Konversation. In: Meggle, Georg (Hg.): Handlung, Kommunikation, Bedeutung. Frankfurt a. M.: Suhrkamp. S. 243-265.

Hawkins, John A. (1994): A performance theory of order and constituency. Cambridge: University Press. (Cambridge studies in Linguistics 73).

Jacobs, Joachim (1988): Fokus-Hintergrund-Gliederung und Grammatik. In: Altmann, Hans (Hg.): Intonationsforschungen. Tübingen: Niemeyer. (Linguistische Arbeiten 200). S. 89-134.

König, Ekkehard/van der Auwera, Johan (1988): Clause integration in German and Dutch conditionals, concessive conditionals, and concessives. In: Haiman, John/Thompson, Sandra A. (Hg.): Clause combining in grammar and discourse. Amsterdam, Philadelphia: Benjamins. (Typological studies in language 18). S. 101-133.

Konerding, Klaus-Peter (2002): Konsekutivität als grammatisches und diskurspragmatisches Phänomen. Tübingen: Stauffenburg. (Studien zur deutschen Grammatik 65).

Küper, Christoph (1991): Geht die Nebensatzstellung im Deutschen verloren? Zur pragmatischen Funktion der Wortstellung in Haupt- und Nebensätzen. In: Deutsche Sprache 19, S. 133-158.

Pasch, Renate (1983): Untersuchungen zu den Gebrauchsbedingungen der deutschen Kausalkonjunktionen da, denn und weil. In: Linguistische Studien. Reihe A. Arbeitsberichte 104. Untersuchungen zu Funktionswörtern (Adverbien, Konjunktionen, Partikeln). Berlin: Akademie der Wissenschaften. S. 41-243.

Pasch, Renate/Brauße, Ursula/Breindl, Eva/Waßner, Ulrich Hermann (2003): Handbuch der deutschen Konnektoren. Linguistische Grundlagen der Beschreibung und syntaktische Merkmale der deutschen Satzverknüpfer (Konjunktionen, Satzadverbien und Partikeln). Berlin/New York: de Gruyter. (Schriften des Instituts für Deutsche Sprache 9).

Peyer, Ann (1997): Satzverknüpfung - syntaktische und textpragmatische Aspekte. Tübingen: Niemeyer. (Reihe Germanistische Linguistik 178). 
Pittner, Karin (1999): Adverbiale im Deutschen. Untersuchungen zu ihrer Stellung und Interpretation. Tübingen: Stauffenburg. (Studien zur deutschen Grammatik 60).

Schwinn, Horst (1997): Linguistische Sprachkritik: ihre Grenzen und Chancen. Heidelberg: Groos. (Sammlung Groos 65).

Thim-Mabrey, Christiane (1988): Satzadverbialia und andere Ausdrücke im Vorvorfeld. In: Deutsche Sprache 16, S. 52-67.

Zifonun, Gisela/Hoffmann, Ludger/Strecker, Bruno et al. (1997): Grammatik der deutschen Sprache. 3 Bände. Berlin/New York: de Gruyter. (Schriften des Instituts für Deutsche Sprache 7). 